\title{
Training Improvement through Subjective Work Analysis: The Example of Radial Puncture
}

\author{
Philippe Fauquet-Alekhine ${ }^{1,2,3}$, Guillaume Bouhours ${ }^{4}$, Justin Texier ${ }^{5}$, Amaury Loret ${ }^{5}$, \\ Saadi Lahlou ${ }^{1} \&$ Jean-Claude Granry ${ }^{5}$ \\ ${ }^{1}$ SEBE-Lab, Department of Psychological \& Behavioural Sc., London School of Economics and Political Science, \\ London, UK \\ ${ }^{2}$ Lab. for Research in Science of Energy, Doué, France \\ ${ }^{3}$ INTRA Robotics, Nuclear Power Plant of Chinon, Avoine, France \\ ${ }^{4}$ Anaesthesia \& Resuscitation Dept., University Hospital of Angers, France \\ ${ }^{5}$ Medical Training Centre, University Hospital of Angers, France \\ Correspondence: Philippe Fauquet-Alekhine, INTRA Robotics, Nuclear Power Plant of Chinon, BP61, 37420, \\ Avoine, France. Tel: 33-24-798-6505.
}

Received: November 13, 2020 Accepted: January 14, 2021 Online Published: January 25, 2021

doi:10.5539/gjhs.v13n3p59 URL: https://doi.org/10.5539/gjhs.v13n3p59

\begin{abstract}
The aim was to test a method developed in nuclear industry applied to a simple activity, the radial artery puncture, and to assess its capacity to improve performance. The method involved digital ethnography based on the Square of PErceived ACtion model applied in real operating situation and first-person perspective video for post-analysis of the activity in order to improve training design. Two types of training sessions were compared in terms of trainees' performance, one of them taking benefits of the digital ethnography analysis (restructured session) and the other without (classic session): medical students trained in the anesthesiology department of the university $(N=24)$ were summoned for training in the framework of their university curriculum. Data obtained were used to restructure the training session and to elaborate an evaluation grid to assess performance in both sessions. Trainees' motivation was assessed through the Motivated Strategies for Learning Questionnaire. The restructured session showed significantly higher overall performance (increased by 13\%), improvement of every criterion assessed and no alteration of motivation. The improvement obtained for radial puncture matches this observed in nuclear industry. The improvement is two folds: at the level of the training efficiency and at the level of trainees' performance.
\end{abstract}

Keywords: assessment, education; radial puncture; simulation; training

\section{Introduction}

Despite the means and care taken designing and conducting training sessions undertaken at the Medical Training Center of of Angers (France) for future residents in anesthesiology at the University Hospital, the results in terms of job performance after training sessions of basic activities were not satisfactory. Simulation training was identified as an efficient means to help students mastering these techniques (Yunoki \& Tetsuro, 2018; Forrest \& McKimm, 2010; Mordi et al., 2018). A first diagnosis led to the conclusion that this was linked with the structure and content of the training sessions, designed through a descriptive method based on training needs identified by experienced professionals as done elsewhere (Steadman et al., 2018). A more incisive method regarding the definition of training needs was sought.

A careful definition of training needs for health simulation training is acknowledged for a long time (World Health Organization, 2010; Holland, 2012) although this point is sometimes neglected (e.g. Flannery \& Zahorsky, 2014) or not correctly defined (e.g. Chatterjee \& Corral, 2017; Persson, 2017). Recently, Persson's review (2017: 315) complained that technical advances have promoted technology at the expense of grounded methods.

To deal with this sort of issue, it was decided to experiment a method recently developed in the nuclear industry, dedicated to the improvement of training programs. This method aims at analyzing work activity through digital ethnography: it uses a miniaturized camera worn at the eye-level (subcam) recording first-person perspective video 
of the subjects' activity in real operating situations. This is followed by an interview based on the Square of PErceived ACtion model or SPEAC model (Fauquet-Alekhine, 2016a, 2020; Fauquet-Alekhine \& Lahlou, 2017) involving workers in a reflexive analysis of their activity. The so-called "SPEAC method" provides a refined description of training needs (matrix of competencies) necessary to perform the activity and is used to develop teaching and training. This method has shown its capacity to identify more fields of competencies (explicit and especially tacit) for the studied activity when compared to other methods (Fauquet-Alekhine, 2016a, 2020). In that it was considered as more incisive than the descriptive method applied in the present case.

The activity chosen for the test was radial artery puncture (to provide a sample of blood for the arterial blood gas test for example), identified as needing improvement (as observed elsewhere: Tavakol et al., 2012; Hernández-Padilla et al., 2016a, 2016b) and presenting the advantage of being simple and short, limiting the cost of the experiments.

The aim of the study was to assess to which extent the SPEAC method would improve simulation training efficiency and thus trainees' performance for radial puncture. Such improvements would contribute to reduce patients' pain, complications and related costs. This was undertaken in the perspective of applying the method to more complex activities with the same objectives.

\section{Method}

\subsection{Design}

The research was led by a Behavioral Psychologist of the SEBE-Lab/Dept. of Psychological \& Behavioural Sc. of the LSE (UK) and the head manager of the Health Simulation Training Center in 2017, and was conducted as follows:

1) An experienced anesthesiologist performed radial puncture in real operating situation and the activity was analyzed using the SPEAC method.

2) A matrix of competencies (list of knowledge and know-how necessary to perform radial puncture) was elaborated on the basis of the analysis of the activity, used to define the training needs and to design an evaluation grid for job performance assessment as done elsewhere (e.g. Hernández-Padilla et al., 2016b).

3) The training session was restructured based on the matrix of competencies. For easier understanding, the training session existing before conducting the research is called "classic session" in the following; the training session obtained after using results of the matrix of competencies is called "restructured session",

4) Classic session and restructured session were then compared in terms of performance with two groups of trainees.

\subsubsection{Analysis of the Work Activity}

Analysis of the radial puncture in real operating situation involved a volunteer anesthesiologist.

The SPEAC method applied for work activity analysis was developed, assessed and validated for workers in nuclear power plants (Fauquet-Alekhine, 2016a, 2020; Fauquet-Alekhine and Lahlou, 2017). It is based on the SPEAC model presenting four interacting poles describing competencies in action (Figure 1). It supports the fact that four poles are required for competencies to be put successfully in action: Having to act, Knowing to act, Wanting to act and Being able to act.

Having to act is a result of the order (manager, patient) and the pre-definition of the task. Knowing to act is that the worker will know to implement whilst performing the task; this pole is a result of learning, of professional training and experience. As it refers to knowledge and know-how, the wording used for the pole is Knowing to act. Should it have only referred to know-how and experience, the wording would have been Knowing how to act. Wanting to act refers to the workers's personal commitment and motivation. Being able to act reflects logistical resources and material means, social conditions and work organization and also the subjects' capacities from the physiological and psychological standpoints. 


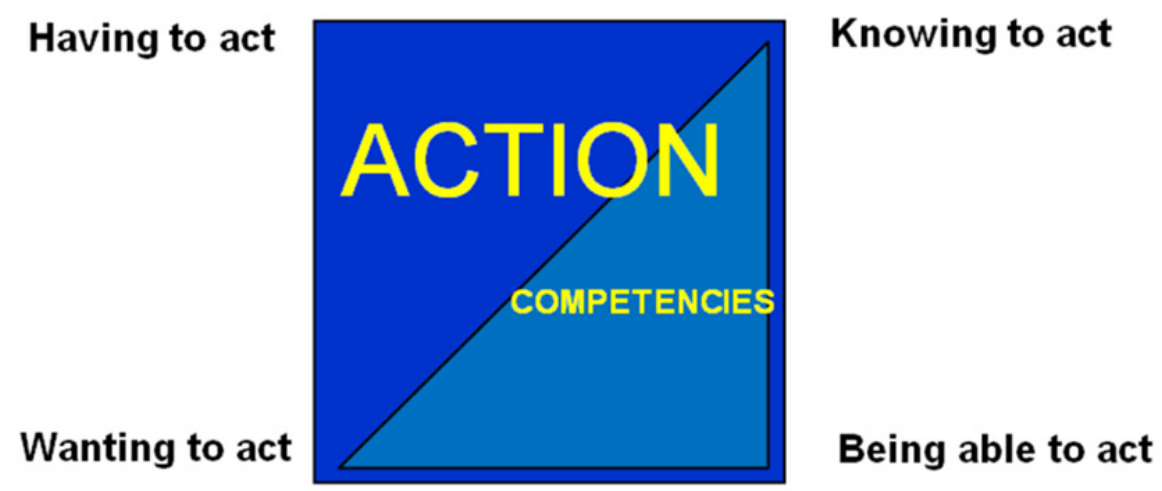

Figure 1. The Square of PErceived ACtion model (SPEAC model)

The schema of the SPEAC method was the following:

- Before undertaking the activity, the anesthesiologist was informed of the aim of the study so that to sign an informed consent, carried out a risk assessment (Fauquet-Alekhine, 2016b; Fauquet-Alekhine et al., 2018) regarding the use of subcam and was equipped with it (preparation phase, $5 \mathrm{~min}$.).

- The anesthesiologist performed his activity which was video recorded from the first-person perspective by means of a subcam worn at the eye-level (capture phase, about $20 \mathrm{~min}$ ).

- After the activity, the subjective video was pre-analyzed by the Behavioral Psychologist to select video relevant sequences.

- The anesthesiologist was met individually by the Behavioral Psychologist to undertake the interview (analysis phase) based on self-confrontation (Von Cranach, 1982; Theureau, 2002), explicitation techniques (Vermersch, 2006) and SPEAC model (Fauquet-Alekhine, 2016a, 2020). A post-analysis of the interview (audio recorded) was undertaken by the Behavioral Psychologist; this provided the output data related to competencies (post-analysis phase).

- Finally, the Behavioral Psychologist and the anesthesiologist discussed resulting output data for validation (validation phase).

The digital equipment used for the first-person recording was made up of three parts linked with cables: i) a micro digital self-powered recorder (size of a mobile phone), ii) a subcam mounted on safety glasses, iii) a lavaliere microphone.

\subsubsection{Matrix of Competencies}

Knowledge and know-how necessary to perform the activity were identified and described through the post-analysis per fields of competencies (the matrix of competencies), one field per column.

For each column of the matrix (Figure 2), knowledge and know-how were listed, regarding both the individual and collective aspects. Knowledge and know-how supposed to be tacit were highlighted in yellow. The first line addressed the fields of competencies designating a general know-how. They were written starting with "knowing how to" followed by an action verb at the infinitive form. So as to simplify the matrix, "knowing how to" was omitted. The individual or collective knowledge referring to information (lessons, books, demonstration...) kept in memory by the anesthesiologist was written using the gerundive form of "to know" followed by direct object. As knowledge fosters know-how (Fauquet-Alekhine, 2020), knowledge was thus to be followed by a goal of action expressed by "in order to know how to" and an action verb at the infinitive form, expressing know-how. However, "in order to know how to" was omitted so as to lighten the matrix. These items were identified during the analysis phase, the validation phase and the post-analysis phase. An excerpt of the matrix is provided on Figure 2. 


\begin{tabular}{|c|c|c|c|c|c|c|c|}
\hline $\begin{array}{l}\text { Fields of } \\
\text { competencies }\end{array}$ & $\ldots$ & $\begin{array}{l}\text { Field \#3 } \\
\text { Disinfect }\end{array}$ & $\begin{array}{l}\text { Field \#4 } \\
\text { Take a blood } \\
\text { sample and } \\
\text { compress }\end{array}$ & $\ldots$ & $\begin{array}{l}\text { Field \#9 } \\
\text { Return the } \\
\text { patient to } \\
\text { safety at the } \\
\text { end of the } \\
\text { activity }\end{array}$ & $\begin{array}{l}\text { Field \#10 } \\
\text { Secure the } \\
\text { blood sample }\end{array}$ & $\ldots$ \\
\hline $\begin{array}{l}\text { Individual } \\
\text { Knowledge and } \\
\text { Know-How }\end{array}$ & $\ldots$ & $\begin{array}{l}\text { Knowing the } \\
\text { asepsis criteria for } \\
\text { soaking and } \\
\text { handling the } \\
\text { compresses in an } \\
\text { appropriate } \\
\text { manner to } \\
\text { disinfect a large } \\
\text { area of skin and } \\
\text { disposing the } \\
\text { used compress } \\
\text { elsewhere } \\
\text { Knowing the } \\
\text { liquid integration } \\
\text { capacity of } \\
\text { compresses to } \\
\text { pour in the } \\
\text { amount of } \\
\text { antiseptic needed } \\
\text { to imbibe several } \\
\text { compresses }\end{array}$ & $\begin{array}{l}\text { Knowing the } \\
\text { characteristics } \\
\text { of arterial blood } \\
\text { to recognize } \\
\text { and validate the } \\
\text { puncture in } \\
\text { progress } \\
\text { Knowing the } \\
\text { characteristics } \\
\text { of arterial blood } \\
\text { to compress } \\
\text { immediately } \\
\text { after collection } \\
\text { Knowing the } \\
\text { characteristics } \\
\text { of arterial blood } \\
\text { to make a } \\
\text { compression } \\
\text { bandage using a } \\
\text { large piece of } \\
\text { adhesive } \\
\text { plaster }\end{array}$ & $\ldots$ & $\begin{array}{l}\text { Knowing the } \\
\text { safety } \\
\text { requirements to } \\
\text { put back the } \\
\text { lateral barriers } \\
\text { of the bed } \\
\text { Knowing the } \\
\text { relevant } \\
\text { parameters of } \\
\text { the scope to } \\
\text { possibly re-set } \\
\text { the scope }\end{array}$ & $\begin{array}{l}\text { Knowing the } \\
\text { ergonomics of } \\
\text { the syringe and } \\
\text { the associated } \\
\text { protocol to } \\
\text { close it properly } \\
\text { and possibly } \\
\text { extract the } \\
\text { presence of } \\
\text { excess air } \\
\text { Knowing the } \\
\text { requirements } \\
\text { and the risks of } \\
\text { errors to } \\
\text { immediately } \\
\text { label the bottle }\end{array}$ & $\ldots$ \\
\hline $\begin{array}{l}\text { Collective } \\
\text { Knowledge and } \\
\text { Know-How }\end{array}$ & $\ldots$ & & & $\ldots$ & & $\begin{array}{l}\text { Knowing the } \\
\text { requirements of } \\
\text { arterial blood } \\
\text { gas test to } \\
\text { quickly transmit } \\
\text { the sample to } \\
\text { the right person }\end{array}$ & $\ldots$ \\
\hline
\end{tabular}

Figure 2. Excerpt of the matrix of competencies resulting from the radial puncture analysis

Note. Highlighted in yellow $=$ tacit knowledge and know-how .

\subsubsection{The Training Sessions}

The sequences of the classic and restructured training sessions (two conditions of the experiment) were identical and carried out with the same simulators (Figure 3): introduction, theoretical lecture, simulation training, simulation debriefing (Fanning and Gabba, 2007; Fauquet-Alekhine and Boucherand, 2016), assessment in practice, session debriefing.

For the restructured session, only the content, the structure of this content and the way it was discussed were adjusted.

The whole sessions lasted $90 \mathrm{~min}$. 


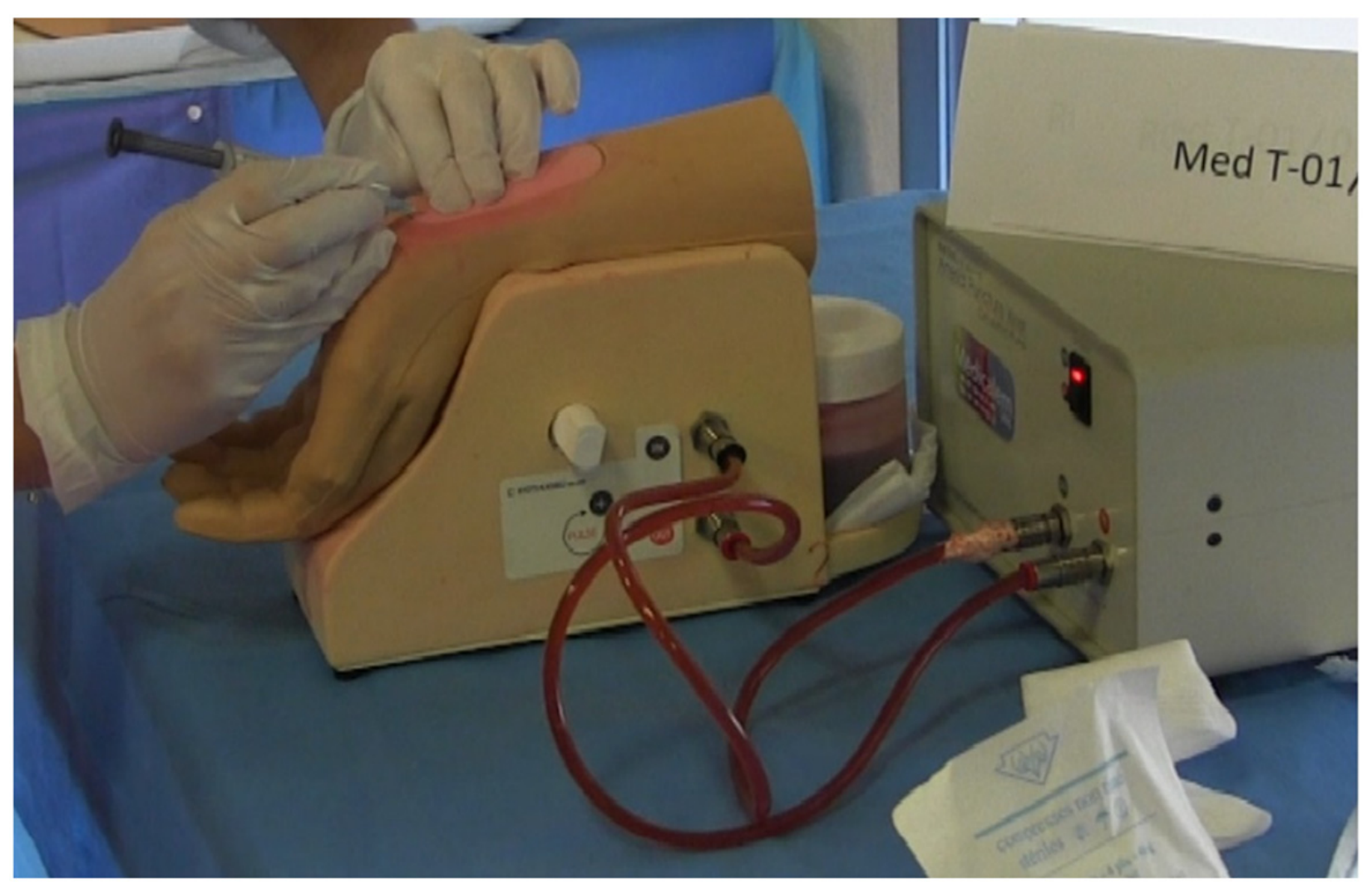

Figure 3. Trainee on simulator for radial puncture. Source: SimS-Med T01-010 ( $\mathrm{t}=04: 48)$

The simulator was an Arterial Puncture Wrist simulator from Kyoto Kagaku Co. (Japan) and has already given evidence for clinical proficiency for such simulation training sessions (Hernández-Padilla et al., 2016a).

\subsubsection{Classic Session and Restructured Session Comparison}

Trainees were summoned to attend the training sessions. It was a blind experiment with two conditions: the classic session and the restructured session. The dependent variable was the trainees' performance, assessed using the evaluation grid. Two independent variables were controlled because they were fixed by the choice of trainees' profile: academic background (medical students in fourth year) and age. The other independent variables were gender, past simulation training, past professional experience, and type of simulation training (classic or restructured). Gender was partly controlled by making an equal proportion in samples of trainees. The effect of past simulation training and past professional experience was assumed to be not significant in first approach (trainees never trained for radial puncture), subject to confirmation through appropriate statistical tests of experimental data. The influence of the two values of the independent variable "type of simulation training" was analyzed. Trainees' performance assessment (dependent variable) was undertaken at the end of each training session.

The training team was a physician-trainer and two Referent residents.

\subsection{Sample/Participants}

Regarding the analysis of a radial puncture performed in real operating situation, an experienced anesthesiologist (male, 41 years old and 13 years of experience in the position) was volunteer.

Regarding the two-condition experiment, trainees were volunteer medical students in fourth year trained in anesthesiology department of the university in view to adopt Anesthesiology specialty ( $N=24$; Table 1$)$, summoned for training in the framework of their university curriculum. 
Table 1. Trainees' characteristics for radial puncture training sessions

\begin{tabular}{llll}
\hline & Classic training session & Restructured training session & All \\
\hline Gender (\% male) & 25 & 25 & 25 \\
Age (y) & 22.5 & 21.0 & 21.75 \\
Experience (y) & 4 th year & 4 th year & 4 th year \\
Number of subjects & 12 & 12 & 24 \\
\hline
\end{tabular}

\subsection{Data Collection}

On the basis of the matrix of competencies, an evaluation grid was elaborated, taking into account specifications of the simulator, the duration of the training session and the trainees' profile (novices). Evaluation criteria concerned clinical examination, technical skills during procedure application, compliance to procedure and communication with patient, assessed on a binary scale as done elsewhere (e.g. Tang et al., 2006). The items were weighted by coefficients (from 1 to 3 ) according to their importance for the activity; the maximum possible score was 68 . The performance of each session was given by the averaged trainees' score.

The validity of the evaluation grid was assessed individually by three experts as done by others (Bausch et al., 2013) on the basis of three statements applied to each item of the grid and evaluated on a five-level Likert scale (strongly disagree, disagree, neither agree nor disagree, agree, strongly agree):

- Relevancy: this item is relevant to assess performance.

- Observability: this item is observable whilst trainee performs the activity.

- Binary assessment feasibility: this item is easy to assess on a binary scale (done, not done).

Job performance assessment of trainees was performed by a physician-trainer of the Medical Training Centre and the Behavioral Psychologist viewing the video recording of their performance.

Immediately after each session, trainees were asked to fill in a form for socio-demographic data and motivation assessment.

The motivation assessment was made up of three scales of the Motivated Strategies for Learning Questionnaire (MSLQ; Pintrich et al., 1991): the Extrinsic Goal Orientation scale (degree to which participants perceived themselves engaged in a task due to grades, rewards, performance, evaluation by others, and competition), Task Value scale (participants' evaluation of how interesting, important and useful the talk was), the Self-Efficacy for Learning and Performance scale (expectancy for success) and self-efficacy. These scales were chosen among the six motivation scales because of their relevance regarding the experiment; the remaining scales dealing with long term academic courses or anxiety were not selected as non-relevant. Two recent in-depth analyses confirmed the reliability of the MSLQ (Kivinen, 2003; Taylor, 2012).

\subsection{Ethical Considerations}

Informed consents were obtained from subjects after informing them of the general purpose of the study.

This study received ethical approval (code of approval: DSP/RS/PFA-4) of the Ethics Committee of the Dept. of Social Psychology (LSE, London, UK) and has therefore been performed in accordance with the ethical standards laid down in the 1964 Declaration of Helsinki.

\subsection{Data Analysis}

Performance scores were calculated per trainee and averaged per cohort. Improvement from one training condition to the other was examined through a t-test of Student. The consistency of the experts' assessment of the evaluation grid and the consistency of the cohorts' answers were evaluated through Cronbach coefficients. Sample size estimation $N_{e s t}$ for a cohort was calculated in the aim of comparing two mean performances. For a confidence interval of 0.05 , a statistical power of 0.80 , a difference between mean performances expected at $3 \%$ of the maximum score (i.e. 2) and a standard deviation expected at 5, we found $N_{e s t}=10.80$. Each group was sized at $N=12$. The influence of the independent variable "past simulation experience" and "past professional experience" was assessed through Pearson correlation coefficient, a $\chi^{2}$-test and a Kolomogorov-Smirnov test. 


\section{Results}

\subsection{Subjects}

The final number of participants was $N=23$ : one of the restructured session subjects was rejected due to a lack of commitment observed by trainers and researchers, objectified by MSLQ data and resulting in the lowest performance score of the cohort.

\subsection{Work Activity Analysis}

The analysis of the work activity led to enhance five points during training and provided a 28 -item evaluation grid used to assess the trainees' performance (see Appendix).

\subsection{Motivation}

The motivation scales being assessed on a Likert scale from 1 to 7 , we found that all subjects presented a score for each selected motivation scale higher than the middle value 3.5 (Table 2) except for the rejected subject. The Cronbach coefficient was $\alpha=0.69$ for the first sample of subjects and 0.80 for the second denoting a good consistency of the answers. The MSLQ scores (individual and averaged per sample) showed an effective commitment of the subjects.

Table 2. Results of motivation self-assessment using MSLQ

\begin{tabular}{|c|c|c|c|c|}
\hline $\begin{array}{l}\text { Session type } \\
\downarrow\end{array}$ & Motivation scale $\rightarrow$ & $\begin{array}{c}\text { Extrinsic Goal } \\
\text { Orientation }\end{array}$ & $\begin{array}{c}\text { Task } \\
\text { value }\end{array}$ & $\begin{array}{c}\text { Self-Efficacy for Learning } \\
\text { and Performance }\end{array}$ \\
\hline \multirow{3}{*}{ Classic session } & $\begin{array}{l}\text { Proportion of subjects over the } \\
\text { middle value } 3.5 \text { of the MSLQ }\end{array}$ & & & \\
\hline & (range: $1-7)$ & $100 \%$ & $100 \%$ & $100 \%$ \\
\hline & $\begin{array}{l}\text { Mean score of all subjects on } \\
\text { MSLQ }\end{array}$ & 4.79 & 5.40 & 5.41 \\
\hline \multirow{3}{*}{$\begin{array}{l}\text { Restructured } \\
\text { session }\end{array}$} & $\begin{array}{l}\text { Proportion of subjects over the } \\
\text { middle value } 3.5 \text { of the MSLQ }\end{array}$ & & & \\
\hline & (range: $1-7$ ) & $100 \%$ & $100 \%$ & $100 \%$ \\
\hline & $\begin{array}{l}\text { Mean score of all subjects on } \\
\text { MSLQ }\end{array}$ & 4.75 & 5.00 & 5.56 \\
\hline
\end{tabular}

No significant correlation was identified between performance and motivation scales. No significant influence on motivation was identified neither from gender nor from age.

\subsection{Performance}

Table 3 gives the comparative performance results with scores averaged per cohort (maximum possible score: 68).

Table 3. Performance results for the classic and the restructured "radial puncture" sessions

\begin{tabular}{|c|c|c|c|c|c|}
\hline Session type & mean score & \% of max score & $\%$ scores $>90 \%$ max score & SD & SD/mean score (\%) \\
\hline Classic session & 55.50 & 81.62 & 16.67 & 6.05 & 10.90 \\
\hline Restructured session & 63.09 & 92.78 & 88.89 & 2.75 & 4.35 \\
\hline
\end{tabular}

Results showed an acceptable consistency for subjects' performance with a Cronbach coefficient $\alpha=0.72$.

The variable "past simulation experience" had no influence on performance as it was zero for all trainees (no previous simulation training). Regarding the variable "past professional experience", the distribution of the number of times the trainees dealt with radial puncture as observers in real operating situation was drawn for each cohort. It was varying from 0 to 6 for trainees of the classic session and 0 to 7 for the other. Kolmogorov-Smirnov test and $\chi^{2}$-test showed that distributions were similar: resp. $D(N=5)=0.15$ and $\chi^{2}(\mathrm{df}=3)=0.58$. The correlation coefficients between the variables "past professional experience" and "performance" were not significant 
$(r(\mathrm{df}=21)<0.27)$, validating the assumption of absence of influence made in first approach.

The correlation coefficients between "performance" and the other variables (age, gender) were not significant $(r(\mathrm{df}=21)<0.24)$

A significant improvement in the performance of the restructured session was found when compared with the classic session:

- $\quad$ average score increased: $+13,7 \%(t(\mathrm{df}=21)=3.98 ; p<0.001)$,

- $\quad$ standard deviation was divided by 2 ,

- trainees whose score was over $90 \%$ of the maximum score were 5 times as many.

Furthermore, two points are of interest: i) the items achieved with $100 \%$ success in the classic session were achieved at the same level in the restructured session, ii) the score of the other items were higher in the restructured session.

\section{Discussion}

\subsection{Performance}

To summarize the results from the classic session to the restructured session for this pilot study:

- Significant improvement in the performance of the restructured session was obtained: increased score by more than $13 \%(p<0.001)$,

- Same motivation score was observed on MSLQ,

- Average performance score was increased for the restructured session with a standard deviation divided by 2 and a percentage of trainees whose score was over $90 \%$ of the maximum score was increased by a factor of 5 .

- Knowledge and know-how that need improvement were clearly identified through the evaluation grid. For example, the SPEAC method pointed out that patient's consent and identity had to be enhanced during training: these points were improved; furthermore, some points not emphasized were improved too: striking successfully at once and the fluidity of gestures. The restructured session was thus more efficient overall than the classic session.

Regarding the contribution of the work analysis, the SPEAC method undoubtedly enriched the content and the structure of the training session. It helped to describe competencies in action as an interacting system of four poles (Figure 1) required for competencies to be put successfully in action: Having to act, Knowing to act, Wanting to act and Being able to act. Therefore, the SPEAC method contributed to give a refined description of the knowledge and know-how than that of the descriptive method. This finding confirmed previous results (Fauquet-Alekhine, 2016a, 2020; Fauquet-Alekhine \& Lahlou, 2017).

\subsection{Limitation}

Results show that however a few points remain to be improved. Some of these residual issues might be corrected in future training sessions using a full-scale simulator with an actor as patient; this might contribute to enhancing the relationship physician-patient and would contribute to force the improvement of the physician-patient exchanges including identification and consent.

Another limitation addresses the evaluation levels of the training session. This limitation is discussed on the basis of the Kirkpatrick four-level pattern (Kirkpatrick \& Kirkpatrick, 1994, 2005, 2007): it is commonly applied to evaluate training programs at different stages (e.g. Bell \& Marais, 2015): trainees' satisfaction, learning, behavior and results (performance in real operating situation). In the present study, the first level was assessed qualitatively through the debriefing undertaken at the end of each training session. The second level was assessed through job performance assessment using the 28 -item grid. The third and fourth levels were partly assessed. While behavior and performance were assessed on simulator, it was not evaluated in real operating situation. This was not possible because trainees were from different parts of the region; therefore, the assessment in real operating situation would have implied a lot of trips on the road for a medical expert in charge of the assessments. Even if assigned to local experts, there was a high probability that they would not be available at the moment the radial puncture is performed.

Finally, being a pilot study, its results should not be generalized without further considerations.

\section{Conclusion}

The aim of the study was to assess to which extent the SPEAC method would improve simulation training efficiency and thus trainees' performance for radial puncture. The results have shown its possibilities to improve 
training session in terms of trainees' performance (more than $13 \%$ for radial puncture) at exit of the simulation: all the criteria assessed were improved when compared to those obtained after using a descriptive method for the design of the training session. The method shows it is a possible solution to deal with medical problems encountered during radial artery puncture in hospital and thus reduce the subsequent economic implications (Wade et al., 2015) when considering the significant quality improvement of the medical act. Application to other medical acts must be considered.

\section{Acknowledgements}

The authors thank all participants from the University Hospital of Angers (France) for their contribution. The authors thank Dr. S. Le Bellu and other members of the Dept. of Psychological \& Behavioural Sc., London School of Economics \& Political Science (LSE, London, UK) for help, advice and cooperation. The authors thank Active Media Concept (www.amc-tec.com) for efficient technical support.

\section{Competing Interests Statement}

The authors declare no conflict of interest of any kind.

\section{References}

Bausch, G., Adermann, J., Andrack, B., Dengl, M., Handwerk, J., Müller, M., ... \& Meixensberger, J. (2013). Design and development process of a next-generation training system for spinal surgery. Simulation, 89(12), 1436-1441. https://doi.org/10.1177/0037549713496151

Bell, J. S., \& Marais, D. (2015). Participatory Training in Monitoring and Evaluation for Maternal and Newborn Health Programmes: Evaluation of a Training Programme Conducted in Africa and South-East Asia. Global journal of health science, 7(2), 192-202. https://doi.org/10.5539/gjhs.v7n2p192

Chatterjee, D., \& Corral, J. (2017). How to write well-defined learning objectives. The journal of education in perioperative medicine: JEPM, 19(4), 1-4. https://doi.org/10.46374/volxix-issue4-chatterjee

Fanning, R. M., \& Gaba, D. M. (2007). The role of debriefing in simulation-based learning. Simulation in healthcare, 2(2), 115-125. https://doi.org/10.1097/SIH.0b013e3180315539

Fauquet-Alekhine, Ph. (2016a). Subjective ethnographic protocol for work activity analysis and occupational training improvement. Brit J Appl Sci Tech, 12(5), 1-16. https://doi.org/10.9734/BJAST/2016/21632

Fauquet-Alekhine, Ph. (2016b). Risk assessment for Subjective Evidence-Based Ethnography applied in High Risk Environment. Adv Res, 6(2), 1-13, Article no.AIR.21597. https://doi.org/10.9734/AIR/2016/21597

Fauquet-Alekhine, Ph. (2020). Knowledge Management in high-risk industries: Coping with skills drain. London: Palgrave Macmillan. https://doi.org/10.1007/978-3-030-49213-7

Fauquet-Alekhine, P., \& Boucherand, A. (2016). Innovative debriefing protocol for simulation training improvement. British Journal of Education, Society \& Behavioural Science, 13(3), 1-13. https://doi.org/10.9734/BJESBS/2016/22418

Fauquet-Alekhine, P., \& Lahlou, S. (2017). The Square of PErceived ACtion model (SPEAC model) applied in digital ethnography for work activity analysis: performance and workers' perception. Current Journal of Applied Science and Technology, 22(3), CJAST-34985. https://doi.org/10.9734/CJAST/2017/34985

Fauquet-Alekhine, Ph., Le Bellu, S., Buchet, M., Berton, J., Bouhours, G., Daviet, Fr., ... \& Lahlou, S. (2018). Risk Assessment for Subjective Evidence-based Ethnography Applied in High Risk environment: Improved Protocol. Adv Res, 16(3), 1-15. https://doi.org/10.9734/AIR/2018/43259

Flannery, M. T., \& Zahorsky, S. (2014). Simulation in residency training: A review. Creative Education, 5(01), 31-35. https://doi.org/10.4236/ce.2014.51007

Forrest, K., McKimm, J. (2010). Using simulation in clinical education. Br J Hosp Med, 71(6), 345-349. https://doi.org/10.12968/hmed.2010.71.6.48454

Hernández-Padilla, J. M., Granero-Molina, J., Márquez-Hernández, V. V., Cortes-Rodriguez, A. E., \& Fernández-Sola, C. (2016a). Effects of a simulation-based workshop on nursing students' competence in arterial puncture. Acta Paul Enferm, 29(6), 678-85. https://doi.org/10.1590/1982-0194201600095

Hernández-Padilla, J. M., Granero-Molina, J., Márquez-Hernández, V. V., Suthers, F., \& Fernández-Sola, C. (2016b). Development and psychometric evaluation of the arterial puncture self-efficacy scale. Nurse education today, 40, 45-51. https://doi.org/10.1016/j.nedt.2016.02.008 
Holland, R. (2012). Designing and implementing training programs. In WHO (Ed.) Managing Access to Medicines and Health Technologies (3rd ed., chapter 52). Geneva: World Health Organization.

Kirkpatrick, D. L., \& Kirkpatrick, J. D. (1994). Evaluating Training Programs. Oakland: Berrett-Koehler Publishers.

Kirkpatrick, D. L., \& Kirkpatrick, J. D. (2005). Transferring Learning to Behavior. Oakland: Berrett-Koehler Publishers.

Kirkpatrick, D. L., \& Kirkpatrick, J. D. (2007). Implementing the Four Levels. Oakland: Berrett-Koehler Publishers.

Kivinen, K. (2003). Assessing motivation and the use of learning strategies by secondary students in three international schools. Tampere University Press.

Mordi, N. G., Sudlow, M., \& Thomson, R. K. (2018). Learning opportunities in simulation for trainees. Br J Hosp Med, 79(10), 154-156. https://doi.org/10.12968/hmed.2018.79.10.C154

Persson, J. (2017). A review of the design and development processes of simulation for training in healthcare-A technology-centered versus a human-centered perspective. Applied ergonomics, 58, 314-326. https://doi.org/10.1016/j.apergo.2016.07.007

Pintrich, P. R. (1991). A manual for the use of the Motivated Strategies for Learning Questionnaire (MSLQ). Report $\mathrm{n}^{\circ}$ NCRIPTAL-91-B-004. Ann Arbor: University of Michigan, National Center for Research to Improve Postsecondary Teaching and Learning.

Steadman, R. H., Huang, Y. M., Koenig, A., Lee, J., Iseli, M., \& Khan, R. (2018). Design of a Screen-Based Simulation for Training and Automated Assessment of Teamwork Skills. University of California Los Angeles Los Angeles United States.

Tang, B., Hanna, G. B., Carter, F., Adamson, G. D., Martindale, J. P., \& Cuschieri, A. (2006). Competence assessment of laparoscopic operative and cognitive skills: objective structured clinical examination (OSCE) or observational clinical human reliability assessment (OCHRA). World Journal of Surgery, 30(4), 527-534. https://doi.org/10.1007/s00268-005-0157-z

Tavakol, M., Ashraf, S., \& Brener, S. J. (2012). Risks and complications of coronary angiography: a comprehensive review. Global journal of health science, 4(1), 65-93. https://doi.org/10.5539/gjhs.v4n1p65

Taylor, R. (2012). Review of the motivated strategies for learning questionnaire (MSLQ) using reliability generalization techniques to assess scale reliability. Doctoral dissertation, Auburn University.

Theureau, J. (2002). L'entretien d'autoconfrontation comme composante d'un programme de recherche empirique et technologique. Communication aux IIe Journées Internationales des Sciences du Sport, INSEP, Paris. https://doi.org/10.3406/insep.2003.1757

Vermersch, P. (2006). L'entretien d'explicitation. Issy-les-Moulineaux: ESF Editeur.

Von Cranach, M. (1982). Goal-directed action. London: Academic Press.

Wade, R. G., Crawfurd, J., Wade, D., \& Holland, R. (2015). Radial artery blood gas sampling: a randomized controlled trial of lidocaine local anesthesia. Journal of evidence-based medicine, 8(4), 185-191. https://doi.org/10.1111/jebm.12177

World Health Organization. (2010). Increasing complexity of medical technology and consequences for training and outcome of care: background paper 4, August 2010. Geneva: World Health Organization. Retrieved from http://www.who.int/iris/handle/10665/70455

Yunoki, K., \& Tetsuro, S. (2018). The role of simulation training in anesthesiology resident education. J Anesth, 32(3), 425-433. https://doi.org/10.1007/s00540-018-2483-y 


\section{Appendix}

\section{Assessment grid objectifying required items for the assessment of the resulting competencies of medical training}

Final version (28 items)

- Selects / Identifies the necessary equipment (especially recognizes the syringe, sterile compresses, alcoholic antiseptic solution)

- Consolidates the identity of the patient: name, bracelet (name, first name and date of birth)

- Requests the consent of the patient

- Explains the purpose of the intervention to the patient

- Performs PHA hand friction (1 pulse) of at least 30 "

- Performs friction before touching compresses or other

- Undertakes a pulse search by applying two or three fingers in the axis of the artery

- Opens the package of compresses (in the right way) to facilitate their imbibition together in the package

- Does not touch compresses

- Puts the antiseptic on the compresses in order to imbibe the whole package

- Threads gloves (normally sterile)

- Disinfects the puncture site with a sterile compress impregnated with antiseptic

- The disinfected area is wide (all the anterior zone of the wrist)

- Ensures that there is no bubble in the syringe

- Re-palpates before stitching and does not lose the indication of the artery during the resumption of syringe (ex: keep a finger in support)

- $\quad$ Spikes in tangential (satisfactory angle: $45^{\circ}$ )

- Spikes in the opposite direction to blood flow

- Strikes successfully at once

- Removes the syringe and protects the needle

- Removes the syringe and locks it with a sterile stopper

- Performs compression of the artery after puncture (prolonged)

- Compresses the artery immediately after puncture

- Places an efficient compressive dressing

- Placement versus patient does not result in physician repositioning

- Placement in relation to the materials does not create any difficulties

- Gestures are fluent (good coordination, good linking)

- Most of the gestures was announced to the patient

- Obtains the sample

\section{Copyrights}

Copyright for this article is retained by the author(s), with first publication rights granted to the journal.

This is an open-access article distributed under the terms and conditions of the Creative Commons Attribution license (http://creativecommons.org/licenses/by/4.0/). 